\title{
PROMOTER ANALYSIS OF MAMMALIAN CLOCK CONTROLLED GENES
}

\author{
KATARZYNA BOŻEK ${ }^{1}$ \\ k.bozek@biologie.hu-berlin.de \\ SZYMON M. KIEŁBASA ${ }^{2}$ \\ kielbasa@molgen.mpg.de \\ ACHIM KRAMER ${ }^{3}$ \\ HANSPETER HERZEL ${ }^{1}$ \\ achim.kramer@charite.de \\ h.herzel@biologie.hu-berlin.de \\ ${ }^{1}$ Institute for Theoretical Biology, Humboldt University, Invalidenstraße 43, D-10115 \\ Berlin, Germany \\ ${ }^{2}$ Max Planck Institute for Molecular Genetics, Ihnestrasse 63-73, 14195 Berlin, Ger- \\ many \\ ${ }^{3}$ Laboratory of Chronobiology, Institute of Medical Immunology, Charité Univer- \\ sitätsmedizin Berlin, Hessische Str. 3-4, 10115 Berlin, Germany
}

\begin{abstract}
The circadian clock is a biological system providing an internal self-sustained temporal framework and adaptation mechanisms to the daily environmental rhythm. One of its behavioral implication in humans is the sleep-wake cycle. The core mammalian circadian clock is a system composed of interacting regulatory feedback loops present in many tissues throughout the body. The core set of circadian clock genes codes for proteins feeding back to regulate not only their own expression, but also that of clock output genes and regulatory pathways. Still, however, our understanding of processes regulated in a circadian fashion and the linkage between the molecular system and behavioral or physiological outputs is poor. Our work aims at identification of clock-controlled genes (CCGs) and their regulatory motifs. We analyzed several microarray measurements of genes with a daily oscillating expression and extracted 2065 of them together with their peak expression phases and oscillation amplitudes. For an in-depth analysis we selected a subset of 167 genes reported by multiple microarray experiments. Gene promoters were scanned in the search for known regulatory motifs of clock genes (E-Box, RRE, D-Box, CRE) as well as other over-represented regulatory motifs. We found an overrepresentation of the E-boxes and D-boxes in the selected subset of 167 CCGs. This over-representation is smaller when the list of 2065 genes is analyzed. The search for other regulatory motifs contained in the TRANSFAC database revealed a strong overrepresentation of some of them such as Sp1, AP-2, STAT1, HIF-1 and E2F. The signals found in the promoter sequences indicate possible regulatory mechanisms important for the coordination of circadian rhythms.
\end{abstract}

Keywords: circadian clock; clock controlled genes; DNA arrays; promoter; transcription factor binding site (TFBS); position specific count matrix (PSCM).

\section{Introduction}

The circadian clock, an internal oscillating system with a period of about 24 hours, is one of the most ubiquitous and preserved biological timing systems. It provides an internal temporal framework and regulates the activities of an organism in relation to environmental cycles. Some of its implication on the human behavior, physiology 
or metabolism are the sleep-wake cycle, hormone rhythms and core body temperature fluctuations.

On the molecular level the mammalian circadian system is composed of interacting positive and negative gene regulatory feedback loops. The key components are the basic helix-loop-helix transcription factors CLOCK and BMAL1 forming a complex that activates the transcription of Per, Cry and RevErb $\alpha$ genes. The PER-CRY protein complex abrogates the transcriptional activity of CLOCK-BMAL1 after translocation to the nucleus. This way the PER and CRY proteins inhibit their own expression. On the other hand, by acting on CLOCK-BMAL1, the complex of PER-CRY indirectly increases its own expression through abolishing the expression of RevErb $\alpha$ - an inhibitor of Bmal1 [1]. Such a system of interconnected positive and negative feedback loops of transcription, translation, protein-protein interaction, phosphorylation, nuclear translocation and protein degradation contributes to delays that create a coordinated molecular cycle approximating the $24 \mathrm{~h}$ environmental period.

The molecular circadian clock is present in many mammalian tissues throughout the body. The core set of circadian clock genes codes for proteins feeding back to regulate not only their own expression, but also that of clock output genes. The clock transcription factors either directly control expression of target genes or indirectly through regulation of other transcription factors.

Studies suggest a hierarchical organization of the circadian regulation in a body with the central pacemaker located in the suprachiasmatic nucleus (SCN) in brain [2]. The master clock is self-sustained and entrained to the daily light/dark cycle. It transmits synchronizing signals to local circadian oscillators in peripheral tissues to achieve and maintain adaptive phase control. Hence, this model of hierarchical circadian oscillators is responsible for regulating the rhythmic outputs observable, e.g., in behavior and physiology.

Although the core molecular pacemaker generating circadian rhythms has been defined both in the SCN and peripheral organs, the molecular outputs that ultimately regulate circadian control of cellular physiology, organ function and behavior are poorly understood. The inter-tissue synchronization mechanism, sustainment of peripheral oscillators or regulation of output pathways by the circadian clock remain an open question. To decipher the interactions among the oscillators and the link between circadian transcriptional output and physiology it is important to study clock-controlled genes, i.e. genes that are under the direct or indirect transcriptional control of the clock transcription factors.

This work aims at identification of cis regulatory motifs of clock-controlled genes. Numerous gene-expression analyses have shown that the mRNA levels of many clock-related genes have high-amplitude circadian oscillations in the SCN or peripheral tissues. Those genes that are not an essential part of the core clock system but reveal circadian oscillations in their mRNA levels are named clock-controlled genes. Recent studies show their importance in mediating local processes within a specific tissue or in such fundamental processes as cell-cycle or metabolism [1]. Investiga- 
tion of the genes and their regulation is a way to come closer to the understanding of the circadian regulation of output pathways in different tissues, synchronization of peripheral circadian oscillators and adaptation to the environmental day-night rhythm.

First, through a literature search, a set of microarray experiments has been found and a list of genes expressed in a circadian manner has been assembled. Next, a promoter analysis of these genes has been performed. We analyze a subset of best annotated genes as well as the full list of genes in the search for known clockregulated motifs. Lastly, a complete search for any over-represented motif from the TRANSFAC database has been completed. We confirm known regulatory motifs such as E-boxes and predict novel binding sites including AP-2, E2F, STAT1, HIF1 and Sp1.

\section{Method and Results}

\subsection{Meta-analysis of microarray experiments}

We performed an extensive literature search in order to find published microarray experiments reporting genes expressed in a circadian manner in mammalian tissues. An initial list of publications [3-13] has been limited to those containing complete gene annotation and full information on gene expression levels and phases.

From the selected papers $[4,5,8,10,12,13]$ a gene list has been assembled. After unification of the overlapping genes and removal of inconsistencies of annotations the list contains 2065 genes expressed in several mouse tissues such as liver, heart, SCN, skeletal muscle and in rat fibroblasts. The overlap among the tissues is limited: 77 genes are expressed in 2 tissues, 23 in 3 and more. The mean deviation of measured peak expression phases of the same gene expressed in two different tissues is striking (e.g. $5.8 \mathrm{~h}$ for the liver-SCN genes, $4.5 \mathrm{~h}$ for liver-heart genes) which is consistent with the observations from other studies [12].

In the first analysis we selected a subset of 167 top-scoring genes that appear in at least three published gene lists. The fact that these genes have been detected by independent experiments indicates that their circadian expression is robust.

\subsection{Promoter analysis}

Following this data search we extracted sequences ranging over $3 \mathrm{kbp}$ upstream and $2 \mathrm{kbp}$ downstream of the transcription start site (TSS) of each gene. The choice of the region has been motivated by previous promoter studies [14-17] detecting clock-related cis elements within several hundred base pairs upstream of a gene TSS up to its first intron. The sequences have been downloaded from EnsEMBL 43 mouse genome [18].

In the search of transcription factor binding sites we used a method for the background model computation and the cut-off threshold estimation for the predicted sites proposed by Rahmann et al. [19] implemented with BioMinerva framework [20]. 
In addition to the basic search in the mouse genome, we performed homology tests by reducing the search space to the conserved parts of promoter sequences of other species orthologous genes (rat, human) as reported by BLASTZ [21].

\subsection{Search for known motifs}

In the first stage of this study we have chosen four cis elements that are known to participate in the circadian regulation: E-boxes, DBP/E4BP4 binding elements (D-boxes), RevErb $\alpha /$ ROR binding elements (RREs) and cyclic AMP responsive elements (CRE) [15, 22]. Position Specific Count Matrices (PSCMs) of the three last motifs have been taken from TRANSFAC version 10.4 [23], whereas the Ebox matrix has been constructed separately basing on eight published experiments on vertebrate tissues [14-17, 24-27]. As suggested in [25] the binding affinity of specific transcription factors to the short 6 bp E-box might be supported by the flanking nucleotides. Hence in the construction of the E-box PSCM 4 bp upstream and downstream of the E-box have been aligned. The sequence logos [28] of the profiles recognized by the four transcription factors are depicted in Fig. 1.
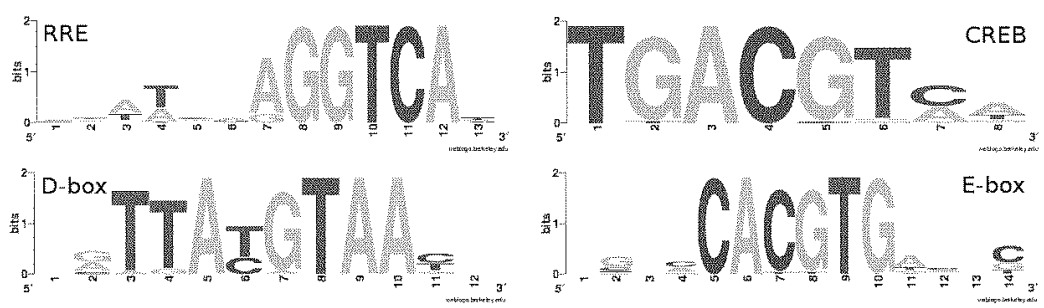

Fig. 1. Sequence logos of known clock-controlled transcription factor binding sites: RevErba/ROR binding element (RRE), cyclic AMP responsive element (CRE), DBP/EABP4 binding element (D-box) and E-box.

First, we counted the number of hits of the known regulatory motifs in the list of 167 selected clock-controlled genes. This number of hits has been compared with the average number of hits obtained for the promoters of sets of genes of the same size (167) sampled 100 times randomly from a subset of 5000 mouse genes not containing those reported as clock-controlled. The promoter region is defined as discussed above. The average number of hits of each motif and the standard deviation of samplings has been calculated. The same sampling of background sequences has been repeated on the full list of 2065 clock-controlled genes. The results of these tests are presented in Table 1.

An over-representation of E-box and D-box motifs can be observed in the promoters of the selected clock-controlled genes as compared to random mouse genes. The differences between the CCGs and random genes exceed 3 standard deviations of the random sampling in the case of E-boxes and nearly 2 standard deviations in the case of D-boxes. The number of motifs in the random samples is approximately 
Table 1. Known clock-controlled transcription factor binding sites predicted in the promoters of 167 selected genes. D-boxes and E-boxes are over-represented in the selected promoters when compared to the predictions done on different background models - a random subset of 5000 mouse genes not containing the CCGs and the full list of 2065 CCGs. Standard deviations of the background samples are given in parentheses.

\begin{tabular}{llccc}
\hline Motif & Consensus sequence & $\begin{array}{c}\text { Hits in } \\
\text { CCGs }\end{array}$ & $\begin{array}{c}\text { Random EnsEMBL } \\
\text { promoters }\end{array}$ & $\begin{array}{c}\text { Full list of } \\
\text { CCGs promoters }\end{array}$ \\
\hline RRE & DNWWNDAGGTCAH & 235 & $227(31.7)$ & $221(14.2)$ \\
CRE & TGACGTMW & 41 & $39(11.8)$ & $50(12.6)$ \\
D-box & NRTTAYGTAAYN & $\mathbf{1 6 2}$ & $\mathbf{1 3 5}(\mathbf{1 7 . 7})$ & $\mathbf{1 2 7}(\mathbf{1 4 . 2})$ \\
E-box & NSNMCACGTGWNNS & $\mathbf{1 6 6}$ & $\mathbf{1 0 1}(\mathbf{1 9 . 3})$ & $\mathbf{1 3 5}(\mathbf{1 8 . 5})$ \\
\hline
\end{tabular}

normally distributed. Thus an excess of motifs in the clock controlled genes of more than 2 standard deviations can be considered as significant. The smaller number of hits in the case of sampling from the full list of CCGs justifies our selection of the subset of 167 genes. In other words, the meta-analysis of multiple microarray experiments enhances the over-representation of E-boxes. Therefore the smaller set of well selected genes allows an easier detection of signals in the promoter analysis. The fact that $\mathrm{CRE}$ and RRE do not appear as over-represented regulatory motifs of the analyzed CCGs might be due to the selection of the genes for this analysis. These motifs as well as D-boxes are known to be relevant for the gene regulatory network of the core oscillator [22] whereas we analyze mainly large sets of output genes.

To validate the relevance of the E-box construction we counted and compared the number of predicted binding sites of another E-box matrix proposed by Kiełbasa et al. [29] as well as the number of canonical E-box sequences (CACGTG) in the CCG and random promoters. Since both approaches lead to equally high scores (data not shown) we consider our PSCM to be well defined and use it in the following tests. Likewise, we performed the site search and counting procedure on the full list of clock-controlled genes. The number of hits was compared to the average number of hits obtained from sampling the same number of genes (2065) from randomly chosen EnsEMBL mouse genes. The results of this test are presented in Table 2.

Table 2. Known clock-controlled transcription factor binding sites predicted in the set of 2065 clock-controlled genes. E-boxes and CRE motifs are considerably over-represented when compared to the predictions done on a random subset of 5000 mouse genes not containing the CCGs. Standard deviations of the background samples are given in parentheses.

\begin{tabular}{llcc}
\hline Motif & Consensus sequence & Hits in CCGs & $\begin{array}{c}\text { Random EnsEMBL } \\
\text { promoters }\end{array}$ \\
\hline RRE & DNWWNDAGGTCAH & 2626 & $2872(104.9)$ \\
CRE & TGACGTMW & 619 & $\mathbf{5 3 3 ( 3 5 . 2 )}$ \\
D-box & NRTTAYGTAAYN & 1566 & $1730(35.2)$ \\
E-box & NSNMCACGTGWNNS & $\mathbf{1 6 3 7}$ & $\mathbf{1 3 0 3 ( 5 8 . 4 )}$ \\
\hline
\end{tabular}


The search for the E-boxes confirms our previous results, however the D-box signal disappears. This might be due to noise in the data as well as different regulatory mechanism of the selected subset of 167 clock-controlled genes.

Fig. 2 contains a schematic illustration of the predicted transcription factor binding sites (TFBSs) and their conservation on the promoter of Per1. All predicted CRE sites and 4 out of 5 predicted E-box sites are confirmed by other studies [16]. The unconfirmed E-box lies outside of the region analyzed by Hida et al. [16].

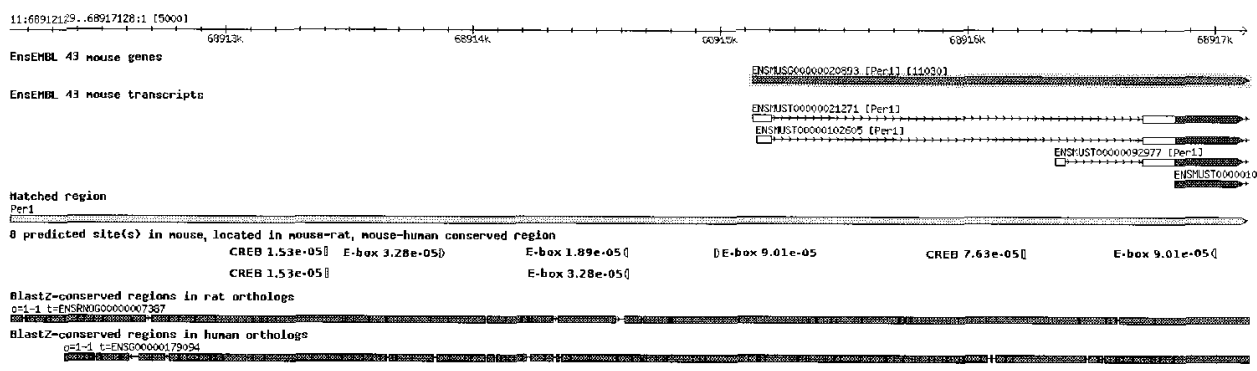

Fig. 2. Predicted conserved TFBSs in the region $3 \mathrm{kbp}$ upstream and $2 \mathrm{kbp}$ downstream of the TSS of Perl mouse gene.

Another example shown in Fig. 3 is the binding site prediction of the RevErb $\alpha$, a gene encoding a member of the nuclear receptor superfamily. This gene is known to be activated by CLOCK-BMAL1 heterodimer binding to E-box sequences and to be regulated through $\mathrm{D}$-boxes and RRE motifs [22, 30, 31]. The E-box predictions in the first intron are in accordance with other studies [31], the co-occurrence of D-boxes could be an indication of a combinatorial regulatory mechanism.

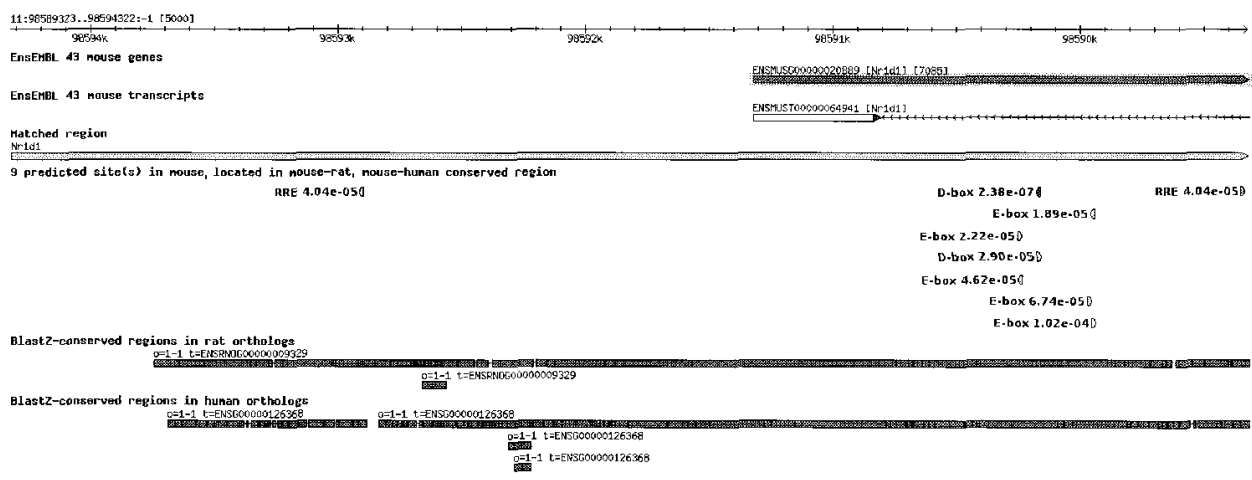

Fig. 3. Predicted conserved TFBSs in the region $3 \mathrm{kbp}$ upstream and $2 \mathrm{kbp}$ downstream of the TSS of RevErb $\alpha$ (Nr1d1) mouse gene. 


\subsection{TRANSFAC motifs search}

Having detected an over-representation of some of the known clock-related transcription factor binding sites, we continued the analysis with the search for an overrepresentation of any TRANSFAC regulatory motif in the clock-controlled gene promoters. All 815 position specific count matrices from the TRANSFAC version 10.4 have been downloaded and the search of the binding sites has been performed on the $5 \mathrm{kbp}$ promoter regions of the subset of 167 CCGs. The number of hits of each of the motifs has been calculated and compared to the average number of hits of the given motif in sampled random mouse gene promoters.

Since this method resulted in a large number of highly over-represented motifs, in the further analysis we considered only those constructed from mammalian experiments and with the number of hits greater than 3 standard deviations above the average number of hits in random genes. The limited list has been clustered according to their sequence similarity [29]. The results of this analysis are shown in the Table 3.

Table 3. A selection of discovered over-represented TRANSFAC binding sites in the 167 selected clockcontrolled genes. Genes presented in the table have the number of hits greater than 3 standard deviations above the average number in random genes. The random genes are sampled from a set of 5000 mouse genes not containing the CCGs. Standard deviations of the background samples are given in parentheses.

\begin{tabular}{lrr|lrr}
\hline Name & $\begin{array}{c}\text { Hits in } \\
\text { CCGs }\end{array}$ & $\begin{array}{c}\text { Random EnsEMBL } \\
\text { promoters }\end{array}$ & Name & $\begin{array}{c}\text { Hits in } \\
\text { CCGs }\end{array}$ & $\begin{array}{c}\text { Random EnsEMBL } \\
\text { promoters }\end{array}$ \\
\hline DEAF1 & 149 & $96(17.4)$ & E2F & 78 & $39(7.8)$ \\
HIC1 & 377 & $214(21.7)$ & E2F-1 & 64 & $46(6.0)$ \\
Pax-4 & 559 & $420(33.3)$ & E2F-1:DP-2 & 64 & $39(5.5)$ \\
VDR & 682 & $534(43.6)$ & GC box & 885 & $616(61.7)$ \\
WT1 & 1098 & $788(81.5)$ & Lmo2 complex & 359 & $279(22.5)$ \\
HES1 & 188 & $136(16.1)$ & LRF & 328 & $235(30.5)$ \\
KROX & 1074 & $721(70.8)$ & MAZ & 983 & $728(52.3)$ \\
Nrf-1 & 229 & $139(25.7)$ & Muscle initiator & 687 & $440(44.0)$ \\
NF-kappaB (p50) & 254 & $162(17.3)$ & MZF & 659 & $540(34.9)$ \\
STAT1 & 159 & $112(14.6)$ & NF-Y & 210 & $153(16.1)$ \\
HNF4 & 1062 & $674(53.1)$ & NRSF & 495 & $391(24.6)$ \\
HIF-1 & 85 & $49(10.3)$ & Sp1 & 1148 & $729(63.9)$ \\
AP-2 & 415 & $247(31.8)$ & SREBP-1 & 314 & $236(17.6)$ \\
AP-2alpha & 426 & $269(31.7)$ & USF2 & 326 & $233(26.8)$ \\
AP-2gamma & 450 & $299(37.4)$ & ZF5 & 245 & $123(21.9)$ \\
CLOCK:BMAL & 165 & $112(13.3)$ & ZNF219 & 1015 & $674(58.1)$ \\
CP2 & 318 & $250(20.9)$ & & & \\
\hline
\end{tabular}

The same comparison test has been done on the promoters of orthologous genes of rat and human. We found a big overlap in the over-representation of motifs in the orthologous gene promoters. Most of the clustered motifs (AP-2, E2F, DEAF1, HES1, HIC1, KROX, MAZ, Muscle initiator, MZF, NF-kappaB, Nrf-1, NRSF, Pax4, Sp1, VDR, WT1, ZF5) show high representation signals in the promoters of clock-controlled genes of rat and human as well.

The over-representation of the CLOCK:BMAL binding site in spite of the high cut- 
off value criteria confirms our previous results and validates our construction of the E-box matrix. Another confirmation is USF2, a motif belonging to the Myc-Max matrix cluster, showing a strong similarity to the E-box sequence [29]. When a cutoff threshold of 2 standard deviations is considered other members of the Myc-Max cluster appear to be over-represented (N-Myc, USF, c-Myc:Max).

To study if the enhancement of certain regulatory motifs is due to the GC content similarity of promoters with the motifs, we calculated the GC ratio of the analyzed promoter regions. The GC content of the regulatory motifs has been obtained from their position count matrices by counting the ratio of $\mathrm{G}$ and $\mathrm{C}$ nucleotides to all nucleotides of sequences used in the matrix construction. Most of the over-represented motifs have a high GC ratio of the composing sequences (e.g. Sp1 - 0.79, GC box - 0.68), significantly above the average ratio of the TRANSFAC matrices $(0.48)$. However even for such a GC-rich motif as Sp1 its over-representation in the predictions is still higher than expected from the ratio of the GC composition of the CCG promoters $(0.49)$ to the one of background sequences $(0.46)$.

\section{Discussion}

The identification of clock-controlled genes and understanding of their regulation is a step towards linking the molecular circadian system with a phenotype. In this study we generated a list of clock-controlled genes based on a set of microarray experiments. We performed promoter analysis in the search for known clock transcription factor binding sites in a selected subset as well as in the full list of CCGs. Having found a strong over-representation of E-boxes and D-boxes we continued with the search for any over-represented regulatory motif included in the TRANSFAC database. We found a set of motifs with a number of hits over 3 standard deviations bigger than in the sampled sets of random mouse genes (Table 3 ).

The predicted participation of the Spl transcription factor in the circadian regulation is consistent with recent biological results. It was shown by electrophoretic mobility shift assays that Sp1 binds certain DNA sequences in a circadian fashion (Hans Reinke and Ueli Schibler, personal communication).

Most of the predicted clock-related transcription factor binding sites have a high GC content. The affinity of the GC-rich cis elements to clock-controlled gene promoters could be therefore a feature specific to the clock-controlled gene regulation.

The approach used can help to identify statistically observable regulatory motifs of this specific gene set. However, due to potential false positives in the TFBS search the predictions on the level of individual genes should be additionally validated.

However, if the predicted transcription factors are a part of the circadian system and show an oscillating transcriptional activity, their analysis can help to reveal biological processes regulated by the circadian system. As an example, the E2F transcription factor family is involved in the control of cell cycle dependent expression of genes that are essential for cellular proliferation. E2F genes may regulate cell cycle progression, apoptosis and suppress cell proliferation [32] which plays an important role in the tumor progression. Revealing such a connection of the circa- 
dian clock to cell cycle and apoptosis might be an important contribution to the chronotherapy of cancer.

Other over-represented binding sites, such as NF-kappaB or STAT1, point to a link between the circadian clock and the immune system. NF-kappaB transcription factors control the expression of multiple genes essential for the immunogenic response and apoptosis [33]. Decoding its relation to the circadian clock might therefore have implications on the therapeutics of a wide variety of human diseases, including immunodeficiency, inflammation, arthritis or cancer.

Another interesting result of our study relates to the transcription factor hypoxia inducible factor 1 (HIF-1). It is a member of the family of transcription factors that respond to changes in available oxygen in the cellular environment. The gene coding for HIF-1 is found to be clock controlled $[10,12]$. As shown in Table 3 , binding sites of this factor are significantly over-represented in the clock controlled genes. Moreover, 9 of 14 target genes included in TRANSFAC are reported to be clock controlled.

Taken together these data may contribute to the understanding of the circadian rhythms in mammals and help to explain regulation of different biological processes. Our further work aims at investigating the specificity of regulation within tissues, and the correlation of the transcription factor binding sites over-representation to the phase of expression peak.

\section{References}

[1] Schibler, U., The daily rhythms of genes, cells and organs. Biological clocks and circadian timing in cells, EMBO Rep., 6:S9-13, 2005.

[2] Yamazaki, S., et al., Resetting Central and Peripheral Circadian Oscillators in Transgenic Rats, Science, 288(5466):682-685, 2000.

[3] Akhtar, R.A., et al., Circadian Cycling of the Mouse Liver Transcriptome, as Revealed by cDNA Microarray, Is Driven by the Suprachiasmatic Nucleus, Curr. Biol., 12(7):540-550, 2002

[4] Duffield, G., et al., Circadian Programs of Transcriptional Activation, Signaling, and Protein Turnover Revealed by Microarray Analysis of Mammalian Cells, Curr. Biol., 12(7):551-557, 2002.

[5] Grundschober, C., et al., Circadian Regulation of Diverse Gene Products Revealed by mRNA Expression Profiling of Synchronized Fibroblasts, J. Biol. Chem., 276(50):46751-46758, 2001.

[6] Humphries, A., et al, cDNA Array Analysis of Pineal Gene Expression Reveals Circadian Rhythmicity of the Dominant Negative Helix-Loop-Helix Protein-Encoding Gene, Id-1, J. Neuroendocrinol, 14(2):101-108, 2002.

[7] Kita, Y., et al., Implications of circadian gene expression in kidney, liver and the effects of fasting on pharmacogenomic studies, Pharmacogenetics, 12(1):55-65, 2002.

[8] Miller, B., et al., Circadian and CLOCK-controlled regulation of the mouse transcriptome and cell proliferation, Proc. Natl. Acad. Sci. USA, 104(9):3342-3347, 2007.

[9] Oishi, K., et al., Genome-wide Expression Analysis of Mouse Liver Reveals CLOCKregulated Circadian Output Genes, J. Biol. Chem., 278(42):41519-41527, 2003.

[10] Panda, S., et al., Coordinated Transcription of Key Pathways in the Mouse by the Circadian Clock, Cell, 109(3):307-320, 2002.

[11] Resuehr, D., Sikes, H., and Olcese, J., Exploratory Investigation of the Effect of Mela- 
tonin and Caloric Restriction on the Temporal Expression of Murine Hypothalamic Transcripts, J. Neuroendocrinol, 18(4):279-289, 2006.

[12] Storch, K.F., et al., Extensive and divergent circadian gene expression in liver and heart, Nature, 417(6884):78-82, 2002.

[13] Ueda, H., et al., A transcription factor response element for gene expression during circadian night, Nature, 418(6897):534-539, 2002.

[14] Leclerc, G. and Boockfor, F., Pulses of Prolactin Promoter Activity Depend on a Noncanonical E-Box that Can Bind the Circadian Proteins CLOCK and BMAL1, Endocrinology, 146(6):2782-2790, 2005.

[15] Chen, W. and Baler, R., The rat arylalkylamine N-acetyltransferase E-box: differential use in a master vs. a slave oscillator, Brain Res. Mol. Brain Res., 81(1-2):43-50, 2000.

[16] Hida, A., et al., The Human and Mouse Period1 Genes: Five Well-Conserved EBoxes Additively Contribute to the Enhancement of mPer 1 Transcription, Genomics, 65(3):224-233, 2000.

[17] Yamaguchi, S., et al., Role of DBP in the Circadian Oscillatory Mechanism, Mol. Cell Biol., 20(13):4773-4781, 2000.

[18] http://www , ensembl.org/Mus \musculus/index.html

[19] Rahmann, S., Möller, T., and Vingron, M., On the Power of Profiles for Transcription Factor Binding Site Detection, Stat. Appl. Genet. Mol. Biol., 2:Article7, 2003.

[20] Kiełbasa, S.M., The BioMinerva framework, in preparation, 2007.

[21] Schwartz, S., et al., Human-Mouse Alignments with BLASTZ, Genome Res., 13(1):103-107, 2003.

[22] Ueda, H., et al., System-level identification of transcriptional circuits underlying mammalian circadian clocks, Nat. Genet., 37(2):187-192, 2005.

[23] http://www.gene-regulation.com/pub/databases.html \\#transfac

[24] Hogenesch, J., et al., The basic-helix-loop-helix-PAS orphan MOP3 forms transcriptionally active complexes with circadian and hypoxia factors, Proc. Natl. Acad. Sci. USA, 95(10):5474-5479, 1998.

[25] Muñoz, E., Brewer, M., and Baler R., Circadian Transcription: thinking outside the E-box, J. Biol. Chem., 277(39):36009-36017, 2002.

[26] Muñoz, E., Brewer M., and Baler, R., Modulation of BMAL/CLOCK/E-Box complex activity by a CT-rich cis-acting element, Mol. Cell Endocrinol, 252(1-2):74-81, 2006.

[27] Jin, X., et al., A Molecular Mechanism Regulating Rhythmic Output from the Suprachiasmatic Circadian Clock, Cell, 96(1):57-68, 1999.

[28] Crooks, G.E., et al., WebLogo: A sequence logo generator, Genome Res., 14(6):11881190, 2004.

[29] Kiełbasa, S.M., Gonze, D., and Herzel, H., Measuring similarities between transcription factor binding sites, BMC Bioinformatics, 6:237-248, 2005.

[30] Chopin-Delannoy, S., et al., A specific and unusual nuclear localization signal in the DNA binding domain of the Rev-erb orphan receptors, J. Mol. Endocrinol, 30(2):197$211,2003$.

[31] Triqueneaux, G., et al., The orphan receptor Rev-erbalpha gene is a target of the circadian clock pacemaker, J. Mol. Endocrinol, 33(3):585-608, 2004.

[32] DeGregori, J. and Johnson, D.G., Distinct and Overlapping Roles for E2F Family Members in Transcription, Proliferation and Apopt, Curr. Mol. Med., 6(7):739-748, 2006.

[33] Liang, Y., Zhou, Y., and Shen, P., NF-kappaB inhibitors for the treatment of inflammatory diseases and cancer, Cell Mol. Immunol., 1(5):343-350, 2004. 